\title{
Determinação dos tipos de resistência em genótipos de feijoeiro ao ataque de Zabrotes subfasciatus (Coleoptera: Bruchidae) ${ }^{1}$
}

\author{
Carolina Prado Batista de Moraes ${ }^{2}$, Arlindo Leal Boiça Junior ${ }^{*}$, Joseane Rodrigues de Souza ${ }^{4}$, \\ Jacqueline Tonielo da Costa
}

\section{RESUMO}

O Zabrotes subfasciatus (Boheman, 1833) (Coleoptera: Bruchidae) é considerado a principal praga do feijão armazenado. O método de controle mais utilizado tem sido o uso de inseticidas; no entanto outros métodos podem ser empregados, como, por exemplo, a resistência de plantas. Este trabalho teve por objetivo determinar os tipos de resistência em 12 genótipos de feijoeiro ao ataque dessa praga. O experimento foi conduzido no Laboratório de Resistência de Plantas a Insetos, do Departamento de Fitossanidade da FCAV\UNESP, Jaboticabal - SP, no período de março a novembro de 2009. Foram realizados testes de não preferência para oviposição, com e sem chance de escolha, e de alimentação, sem chance de escolha. Concluiu-se que os genótipos Dor 391, Dor 476, IAPAR-MD 806 e Raz 59 apresentam resistência do tipo não preferência para oviposição por Z. subfasciatus, em teste com chance de escolha; no teste sem chance de escolha todos os genótipos foram igualmente ovipositados pelo inseto; no teste com chance de escolha, machos e fêmeas foram igualmente atraídos por todos os genótipos testados; e Raz 56, Arc 2, Raz 55, Raz 49 e Raz 59 apresentaram resistência dos tipos não preferência para alimentação e,ou, antibiose.

Palavras-chave: Phaseolus vulgaris, grãos armazenados, resistência de plantas a insetos, caruncho.

\section{ABSTRACT}

\section{Determination of resistance to the attack of Zabrotes subfasciatus (Coleoptera: Bruchidae) in bean genotypes}

Zabrotes subfasciatus (Boheman, 1833) (Coleoptera: Bruchidae) is considered the main stored bean pest. The most widely used method of control is the use of insecticides. However, other control methods may be applied, such as plant resistance. Aiming to find alternative strategies to its control, this study evaluated the types of resistance in 12 bean genotypes. The experiment was carried out at the Laboratory of Plant Resistance to Insects of the Department of Phytopathology of the FCAV/UNESP, Jaboticabal/SP, from March to November 2009. Oviposition non-preference (free choice and no-choice) tests and the no-choice test for feeding were carried out. The results demonstrated that: the genotypes Dor 391, Dor 476, IAPAR-MD 806, and Raz 59 showed non-preference to oviposition by Z. subfasciatus, in the free-choice test; in the no-choice test, all genotypes were equally oviposited by the insect; in the free-choice chance test, males and females were equally attracted by all tested genotypes and Raz 56, Arc 2, Raz 55, Raz 49, and Raz 59 showed non-preference to feeding and/or antibiosis.

Key words: Phaseolus vulgaris L., stored bean, host plant resistance, Mexican bean weevil.

Recebido para publicação em 01/12/2010 e aprovado em 20/07/2011

'Extraído da monografia do primeiro autor, Instituição de Fomento - CNPq.

${ }^{2}$ Graduanda em Agronomia, Universidade Estadual Paulista, Faculdade de Ciências Agrárias e Veterinárias, Departamento de Fitossanidade, Via de Acesso Prof. Paulo Donato Castellane s/n ${ }^{\circ}$, 14884-900, Jaboticabal, SP, Brasil. carolzinham2@ @otmail.com.

3*Engenheiro-Agrônomo - Professor Doutor, Universidade Estadual Paulista, Faculdade de Ciências Agrárias e Veterinárias - FCAVIUNESP, Departamento de Fitossanidade, Via de Acesso Prof. Paulo Donato Castellane s/n, 14884-900, Jaboticabal, SP. aboicajr@fcav.unesp.br . Autor para correspondência.

${ }^{4}$ Engenheira-Agrônoma - Doutoranda, Universidade Estadual Paulista, Faculdade de Ciências Agrárias e Veterinárias - FCAVIUNESP, Departamento de Fitossanidade, Via de Acesso Prof. Paulo Donato Castellane s/n, 14884-900, Jaboticabal, SP. joseaneagro@yahoo.com.br.

${ }_{5}^{5}$ Engenheira-Agrônoma - Mestranda, Universidade Estadual Paulista, Faculdade de Ciências Agrárias e Veterinárias - FCAVlUNESP, Departamento de Fitossanidade, Via de Acesso Prof. Paulo Donato Castellane s/n, 14884-900, Jaboticabal, SP. arrombatonielo@yahoo.com.br. 


\section{INTRODUÇÃO}

O feijoeiro Phaseolus vulgaris L.(Fabaceae) é suscetível ao ataque de inúmeras pragas, e, dentre elas, destacam-se as que atacam os grãos armazenados, conhecidas popularmente como carunchos (Vieira \& Yokoyama, 2000). O caruncho-do-feijão, Zabrotes subfasciatus (Boheman, 1833) (Coleoptera: Bruchidae) é considerado a principal praga do feijão armazenado nas regiões tropicais, podendo também ser encontrado em regiões de clima temperado e frio (Abreu, 2005).

Os danos causados por Z. subfasciatus no armazenamento são consideráveis e decorrentes da penetração e alimentação, somente das larvas, no interior dos grãos provocando perda de peso, redução do valor nutritivo e do grau de higiene do produto, depreciando a qualidade comercial, por presença de insetos, ovos e excrementos (Gallo et al., 2002; Quintela, 2002). De acordo com estes mesmos autores, graças à formação de galerias feitas pelas larvas de Z. subfasciatus, ocorre a destruição dos cotilédones, reduzindo o peso da semente e favorecendo a entrada de micro-organismos e ácaros.

No Brasil, a ocorrência de Z. subfasciatus é generalizada, em todas as regiões produtoras (Vieira \& Yokoyama, 2000). As perdas quantitativas, causadas por pragas no armazenamento, no Brasil, podem resultar em até $10 \%$ do total produzido anualmente. Além disso, existem as perdas qualitativas, que são mais preocupantes, pois podem comprometer totalmente o uso do grão produzido (Lorini, 2008).

A resistência de plantas e o uso de pós vegetais, extraídos de plantas inseticidas, têm sido empregados no controle de Z. subfasciatus em feijoeiro (Procópio et al., 2003). No entanto, o controle químico tem sido o método mais eficaz e o mais usado, em razão das facilidades para aplicação e da maior rapidez de ação (Faroni \& Silva, 2008). O controle químico, porém, apresenta os inconvenientes como o aumento dos custos e os resíduos tóxicos, que podem contaminar o meio ambiente e o homem (Lara, 1991).

Diante do exposto, a resistência de plantas é considerada um método promissor, pois não exige conhecimentos específicos, por parte dos agricultores para a sua utilização, apresenta baixo custo (Lara, 1991), além da ausência de contaminação dos grãos e da compatibilidade com outros métodos de controle (Mazzoneto \& Boiça Júnior, 1999; Mazzonetto \& Vendramim, 2002).

A procura por fontes de resistência em acessos de feijão tem sido alvo de pesquisas, como método de controle ao Z. subfasciatus (Vieira et al., 2005). Schoonhoven \& Cardona (1982), em estudos com mais de 4000 acessos de feijão cultivados, concluíram que os níveis de resistência a Z. subfasciatus eram muito baixos. Schoonhoven et al. (1983) avaliaram formas silvestres de P. vulgaris, de origem mexicana, e constataram linhagens com alto nível de resistência a Z. subfasciatus e Acanthoscelides obtectus (Say, 1831) e que o tipo de resistência associado é do tipo antibiose. No Brasil, vários estudos foram realizados, sendo identificados genótipos de feijão resistentes ao Z. subfasciatus (Pereira et al., 1995; Wanderley et al., 1997; Souza et al., 1997; Mazzonetto \& Boiça Junior, 1999; Boiça Júnior et al., 2002 e Miranda et al., 2002).

Alguns estudos mostraram que a proteína arcelina, armazenada nas sementes e presente somente em linhagens de feijão silvestres, está associada à resistência a $Z$. subfasciatus (Osborn et al., 1986). A proteína arcelina pertence à categoria das lectinas, em que se incluem as fitohemaglutininas e inibidores de alfa-amilase (Paes et al., 2000). Até o momento, sete linhagens, contendo os alelos com a proteína arcelina, que conferem resistência a $Z$. subfasciatus, já foram identificadas e denominadas de arcelina 1 (Arc 1) a arcelina 7 (Arc 7), sendo que as variantes das proteínas arcelina 1 (Arc 1) e arcelina 5 (Arc 5) têmse mostrado as mais promissoras para conferir resistência em plantas leguminosas (Acosta-Galegos et al., 1998).

Dessa forma, estudos que visam à busca por genótipos resistentes à Z. subfasciatus são de suma importância para a cultura do feijoeiro, pois irão proporcionar redução das perdas pós-colheita, causadas pelo referido inseto.

Portanto, o presente trabalho teve como objetivo determinar, em 12 genótipos de feijoeiro, os tipos de resistência ao ataque de Z. subfasciatus.

\section{MATERIAL E MÉTODOS}

A pesquisa foi desenvolvida no Laboratório de Resistência de Plantas a Insetos, do Departamento de Fitossanidade, da Faculdade de Ciências Agrárias e Veterinárias FCAV\UNESP, Jaboticabal, SP. Os insetos utilizados nos experimentos foram oriundos de criação estoque do laboratório, sendo criados em feijão, P. vulgaris, cultivar Bolinha, considerada suscetível (Boiça Junior et al., 2002), em frascos de vidro de cinco litros, fechados com tampas metálicas vazadas e revestidas com tela de náilon. A cada 30 dias, o material era peneirado e os adultos separados para iniciar a infestação de novos frascos. A criação foi mantida em sala climatizada, em temperatura de 27 $\pm 2{ }^{\circ} \mathrm{C}$, umidade relativa de $75 \pm 5 \%$ e fotofase de 12 horas.

Os genótipos avaliados foram: Arc 1, Arc 2, Arc 3, Arc 4, Raz 49, Raz 55, Raz 59, Raz 50, HF 5465, Dor 391, Dor 476 e IAPAR-MD 806. Tais genótipos foram adquiridos junto ao Instituto Agronômico de Campinas - IAC, em quantidades suficientes para a montagem dos experimentos.

\section{Teste com Chance de Escolha}

$\mathrm{O}$ delineamento experimental utilizado foi em blocos ao acaso, em quatro repetições, sendo estas constituídas de amostras de $10 \mathrm{~g}$ de grãos de cada genótipo. O teste foi realizado em arenas constituídas de bandejas circulares de alumí- 
nio, de $30 \mathrm{~cm}$ de diâmetro e $5 \mathrm{~cm}$ de altura, contendo, em seu interior, placas de isopor circulares de 29,4 cm de diâmetro e $2 \mathrm{~cm}$ de altura, com 12 aberturas circulares, com diâmetro de $3,8 \mathrm{~cm}$ nas extremidades, onde acondicionaram-se recipientes plásticos de $3,9 \mathrm{~cm}$ de altura e $3,8 \mathrm{~cm}$ de diâmetro, contendo as amostras de $10 \mathrm{~g}$ de grãos dos genótipos em estudo. Estes potes foram dispostos de forma equidistante do centro da placa, onde foram liberados 84 casais recém-emergidos de Z. subfasciatus, conforme a metodologia descrita por Schoonhoven \& Cardona (1982). Para que não houvesse fuga dos insetos, as bandejas foram fechadas com outra bandeja de alumínio e vedadas com fita adesiva. Após sete dias, as bandejas foram abertas e contados o número de machos, fêmeas e total de Z. subfasciatus atraídos pelos grãos nos genótipos. Dez dias após a abertura, procedeu-se à contagem do número de ovos viáveis nos genótipos com o auxílio de um microscópio estereoscópio e um contador manual, sendo os ovos esbranquiçados considerados viáveis e, os translúcidos, inviáveis.

\section{Teste sem Chance de Escolha}

O delineamento experimental deste teste foi inteiramente casualizado, com cinco repetições. Acondicionaram-se $10 \mathrm{~g}$ de grãos em recipientes plástico de $3,9 \mathrm{~cm}$ de altura e $3,8 \mathrm{~cm}$ de diâmetro, onde foram confinados sete casais recém-emergidos de Z. subfasciatus por amostras, durante sete dias, conforme a metodologia descrita por Schoonhoven \& Cardona (1982). Foram avaliados os seguintes parâmetros: número de insetos emergidos (NIE), percentagem de insetos emergidos (PIE), período de eclosão da larva à emergência do adulto (dias) (PELEA), total de massa seca consumida pelos insetos (mg) (TMSC) e massa seca consumida por inseto (mg) (MSCI).

Sete dias após o confinamento, os adultos foram retirados e dez dias após foi realizada a contagem do número de ovos viáveis, conforme descrito no teste com chance de escolha. Posteriormente, os ovos foram acondicionados em placas de Petri, para se contabilizar o total de insetos emergidos e, assim, calcular-se o percentual de insetos emergidos. A partir do dia da eclosão da larva até a emergência de insetos adultos, foi contabilizado o período de desenvolvimento dos insetos. A aproximadamente 25 dias do confinamento, foram iniciadas observações diárias das amostras de feijão, a fim de se contar e retirar os adultos emergidos. Após o término da emergência dos insetos em todas as amostras, estas foram colocadas em estufa, a $70^{\circ} \mathrm{C}$, para secagem por dois dias e, pela diferença em relação ao peso das alíquotas, foi determinada a massa da matéria seca consumida pelos insetos.

Em ambos os testes, os dados foram transformados em $(x+0,5)^{1 / 2}$ e submetidos à análise de variância $e$, as médias, comparadas pelo teste de Tukey a $5 \%$ de significância.

\section{RESULTADOS E DISCUSSÃO}

\section{Teste com Chance de Escolha}

Os genótipos diferiram significativamente em relação ao número total de ovos viáveis de Z. subfasciatus (Tabela 1). O número total de ovos viáveis depositados por Z. subfasciatus variou entre 121,75 e 223,00, sendo que os genótipos Dor 391, IAPAR-MD 806 e Dor 476 destacaram-se como os menos preferidos, pois apresentaram os menores valores $(120,25 ; 121,75$ e 122,00 ovos viáveis, respectivamente), quando comparados com os genótipos HF 5465 e Arc 2 (223,00 e 212,00 ovos viáveis, respectivamente), que foram os mais preferidos pelo caruncho (Tabela 1). Nesse caso, e considerando-se os resultados para o genótipo Arc 2, os mesmos estão de acordo com Acosta-Galegos et al. (1998) que relataram que, apenas as variantes das proteínas arcelina 1 e 5 dos genótipos Arc 1 e Arc 5 têm-se mostrado como os mais promissores por conferir resistência a Z. subfasciatus em plantas leguminosas. RibeiroCosta et al. (2007) também verificaram maior número de ovos viáveis de Z. subfasciatus no genótipo Arc 2, sendo que este não diferiu da maioria dos genótipos sem arcelina.

Os resultados de número de machos, fêmeas e total de adultos de Z. subfasciatus, atraídos pelos materiais em estudo, não mostraram diferenças significativas, sugerindo pouca interferência na oviposição do inseto (Tabela 1). Para Lara (1997), os genótipos Arc 1 e Arc 2 foram igualmente atraídos por adultos de Z. subfasciatus, demonstrando que as variantes das proteínas arcelina, conforme descrito para o número total de ovos, não interfere na atratividade e oviposição de Z. subfasciatus nos genótipos estudados. Boiça Júnior et al. (2002) estudaram o comportamento de genótipos de feijoeiro sem arcelina e, dentre eles, o IAPAR-MD 806 ao ataque de $Z$. subfasciatus e concluíram que todos os genótipos foram igualmente atraídos e ovipositados por fêmeas do caruncho.

Os genótipos portadores de arcelina, possivelmente interferem na alimentação de Z. subfasciatus, sendo essa interferência devida à presença de compostos químicos, pois a arcelina foi considerada responsável pela resistência do tipo antibiose, presente nos tipos silvestres de P. vulgaris, ao caruncho (Osborn et al., 1986). Outras causas da resistência de genótipos de feijão ao Z. subfasciatus foram relatadas por Vieira $e t$ al. (2005) e estão associadas à dureza da testa e ao pequeno tamanho das sementes de tipos silvestres de $P$. vulgaris, como as dos genótipos portadores de arcelina. Além disso, a forma como Z. subfasciatus fixa a postura dos seus ovos nos grãos, não deixando-os soltos, pode ser um indicativo de que esses insetos 
provavelmente selecionam o hospedeiro, pois os insetos apresentam como regra geral o comportamento de ovipositar em substratos que garantam o desenvolvimento das larvas, assegurando, dessa forma, a sobrevivência da espécie (Lara, 1991).

\section{Teste sem chance de escolha}

Os resultados obtidos para o número de ovos viáveis encontrados nos 12 genótipos não apresentaram diferenças significativas entre si, sendo a média geral de 170,23 ovos e o desvio padrão de 18,10 . No entanto, de modo geral, os dados obtidos encontrados neste teste seguem a mesma tendência dos resultados apresentados no teste com chance de escolha, no qual o genótipo Arc 2 apresentou maior número de ovos viáveis $(214,60)$. A não existência de diferenças significativas também foi observada por Miranda et al. (2002), em estudo com genótipos sem e portadores de arcelina de 1 a 4 .

Em relação ao número e percentagem de insetos emergidos, foram observadas diferenças significativas entre os genótipos avaliados (Tabela 2). Foram menos favoráveis à emergência de Z. subfasciatus os genótipos Raz 56 e Raz 59, proporcionando menor número de insetos emergidos. Nesses genótipos e também no Raz 55 e Arc 2 , foi verificado também menor percentual de $Z$. subfasciatus emergidos. Possivelmente, algum fator de resistência esteja associado a esses genótipos. No entanto, nos genótipos HF 5465, Dor 391 e Arc 3 ocorreu efeito inverso, o que levou à maior preferência do caruncho, sendo estes mais favoráveis ao número de insetos emergidos. Em relação ao percentual de Z. subfasciatus emergidos, foi observado maior percentual de emergência nos cultivares Dor 476, IAPAR-MD 806, HF 5465, Dor 391 e Arc 3 (Tabela 2).

Resultados semelhantes foram obtidos por Barbosa et al. (1999) que estudaram o efeito da proteína arcelina na biologia de Z. subfasciatus em feijoeiro. De acordo com os autores, insetos criados nos genótipos Arc 3 e Arc 4 não tiveram sua progênie afetada e, portanto, eles não interferiram no número de ovos viáveis e que o genótipo Arc 2 reduziu o número de insetos emergidos. De acordo com Vieira et al. (2005), o mecanismo responsável pela resistência a $Z$. subfasciatus em feijoeiro é a antibiose, sendo responsável por efeitos adversos na sua biologia e que esses efeitos são provocados pela proteína arcelina, presente nos tipos silvestres de $P$. vulgaris.

Os resultados obtidos para o período da eclosão da larva à emergência do adulto mostraram diferenças significativas (Tabela 2). Os insetos provenientes dos genótipos Arc 1, Arc 2, Arc 3 Arc 4, Raz 55, Raz 56, Raz 49 e Raz 59 apresentaram período de desenvolvimento mais longo, variando de 35,90 a 32,98 dias, sugerindo resistência do tipo antibiose. Opostamente, com menores períodos, e, portanto suscetíveis, encontram-se os genótipos HF 5465, Dor 391, Dor 476 e IAPAR-MD 806 que variou de 29,53 a 28,74 dias. Outros trabalhos também demonstraram o aumento do período de desenvolvimento de $Z$. subfasciatus em genótipos portadores de arcelina, como os obtidos por Wanderley et al. (1997) com o genótipo Arc 1, Miranda et al. (2002) com os genótipos Arc 1 e Arc 4 e Mazzonetto \& Vendramim (2002) com os genótipos Arc1, Arc 2, Arc 3 e Arc 4.

Tabela 1. Número de ovos viáveis em teste com chance de escolha, e de insetos atraídos machos, fêmeas e total de Zabrotes subfasciatus em genótipos de feijoeiro, em teste com chance de escolha

\begin{tabular}{|c|c|c|c|c|}
\hline \multirow{2}{*}{ Genótipos } & \multirow{2}{*}{$\begin{array}{l}\text { Número de } \\
\text { ovos viáveis }\end{array}$} & \multicolumn{3}{|c|}{ Número de insetos atraídos } \\
\hline & & Machos & Fêmeas & Total \\
\hline Arc 1 & $177,25 \mathrm{abc}^{1}$ & $5,75 \mathrm{a}$ & $5,75 \mathrm{a}$ & $11,50 \mathrm{a}$ \\
\hline Arc 2 & $212,00 \mathrm{a}$ & $8,50 \mathrm{a}$ & $8,75 a$ & $17,25 \mathrm{a}$ \\
\hline Arc 3 & $191,00 \mathrm{ab}$ & $4,75 \mathrm{a}$ & $4,75 \mathrm{a}$ & $9,50 \mathrm{a}$ \\
\hline Arc 4 & $163,00 \mathrm{abc}$ & $6,00 \mathrm{a}$ & $5,25 \mathrm{a}$ & $11,25 \mathrm{a}$ \\
\hline Raz 55 & $155,50 \mathrm{abc}$ & $5,50 \mathrm{a}$ & $4,25 \mathrm{a}$ & $9,75 \mathrm{a}$ \\
\hline Raz 56 & $171,00 \mathrm{abc}$ & $6,50 \mathrm{a}$ & $6,75 \mathrm{a}$ & $13,25 \mathrm{a}$ \\
\hline Raz 49 & $168,75 \mathrm{abc}$ & $8,00 \mathrm{a}$ & $6,75 \mathrm{a}$ & $14,75 \mathrm{a}$ \\
\hline Raz 59 & $135,50 \mathrm{bc}$ & $5,75 \mathrm{a}$ & $6,50 \mathrm{a}$ & $12,25 \mathrm{a}$ \\
\hline HF 5465 & $223,00 \mathrm{a}$ & $5,25 \mathrm{a}$ & $6,00 \mathrm{a}$ & $11,25 \mathrm{a}$ \\
\hline Dor 391 & $120,25 \mathrm{c}$ & $6,25 \mathrm{a}$ & $3,00 \mathrm{a}$ & $9,25 \mathrm{a}$ \\
\hline Dor 476 & $122,00 \mathrm{c}$ & $5,00 \mathrm{a}$ & $5,25 \mathrm{a}$ & $10,25 \mathrm{a}$ \\
\hline IAPAR-MD 806 & $121,75 \mathrm{c}$ & $5,00 \mathrm{a}$ & $3,25 \mathrm{a}$ & $8,25 \mathrm{a}$ \\
\hline F (Tratamentos) & $6,89 * *$ & $0,78^{\mathrm{ns}}$ & $1,52^{\mathrm{ns}}$ & $1,44^{\mathrm{ns}}$ \\
\hline Desvio padrão & 33,04 & 1,12 & 1,54 & 2,44 \\
\hline C.V.(\%) & 8,20 & 20,87 & 21,97 & 17,44 \\
\hline
\end{tabular}

${ }^{1}$ Médias seguidas de mesma letra na mesma coluna não diferem estatisticamente a 5\% de significância, pelo teste de Tukey. Para as análises, os dados foram transformados em $(\mathrm{x}+0,5)^{1 / 2}$.** significativo a $5 \%$ de probabilidade; ns não significativo. 
Tabela 2. Número de insetos emergidos (NIE), percentagem de insetos emergidos (PIE), período de eclosão da larva à emergência do adulto (dias) (PELEA), total de massa seca consumida pelos insetos (mg) (TMSC) e massa seca consumida por inseto (mg) (MSCI) de Zabrotes subfasciatus criados em genótipos de feijoeiro, em teste sem chance de escolha

\begin{tabular}{lllccc}
\hline Genótipos & \multicolumn{1}{c}{ NIE $^{2}$} & PIE $^{2}$ & PELEA $^{2}(\mathbf{d i a s})$ & TMSC $^{2}(\mathbf{m g})$ & MSCI $^{2}(\mathbf{m g})$ \\
\hline Arc 1 & $33,80 \mathrm{bcde}^{1}$ & $17,69 \mathrm{bc}$ & $33,49 \mathrm{a}$ & $42,50 \mathrm{abc}$ & $12,58 \mathrm{~b}$ \\
Arc 2 & $28,80 \mathrm{cde}$ & $13,29 \mathrm{c}$ & $35,01 \mathrm{a}$ & $23,54 \mathrm{bc}$ & $7,60 \mathrm{~b}$ \\
Arc 3 & $78,00 \mathrm{a}$ & $43,87 \mathrm{a}$ & $32,98 \mathrm{a}$ & $67,29 \mathrm{ab}$ & $8,26 \mathrm{~b}$ \\
Arc 4 & $54,60 \mathrm{abcd}$ & $35,00 \mathrm{ab}$ & $34,64 \mathrm{a}$ & $55,42 \mathrm{abc}$ & $9,94 \mathrm{~b}$ \\
Raz 55 & $23,60 \mathrm{de}$ & $13,14 \mathrm{c}$ & $35,52 \mathrm{a}$ & $27,81 \mathrm{bc}$ & $5,30 \mathrm{~b}$ \\
Raz 56 & $20,40 \mathrm{e}$ & $10,76 \mathrm{c}$ & $35,90 \mathrm{a}$ & $16,01 \mathrm{c}$ & $10,78 \mathrm{~b}$ \\
Raz 49 & $27,20 \mathrm{cde}$ & $16,39 \mathrm{bc}$ & $34,89 \mathrm{a}$ & $23,99 \mathrm{bc}$ & $8,24 \mathrm{~b}$ \\
Raz 59 & $16,40 \mathrm{e}$ & $13,05 \mathrm{c}$ & $35,76 \mathrm{a}$ & $49,02 \mathrm{abc}$ & $30,78 \mathrm{a}$ \\
HF 5465 & $82,60 \mathrm{a}$ & $50,77 \mathrm{a}$ & $28,74 \mathrm{~b}$ & $85,20 \mathrm{a}$ & $10,34 \mathrm{~b}$ \\
Dor 391 & $88,60 \mathrm{a}$ & $60,86 \mathrm{a}$ & $29,53 \mathrm{~b}$ & $88,82 \mathrm{a}$ & $9,98 \mathrm{~b}$ \\
Dor 476 & $65,20 \mathrm{abc}$ & $42,17 \mathrm{a}$ & $28,81 \mathrm{~b}$ & $79,66 \mathrm{a}$ & $11,82 \mathrm{~b}$ \\
IAPAR-MD 806 & $69,60 \mathrm{ab}$ & $44,62 \mathrm{a}$ & $28,92 \mathrm{~b}$ & $64,20 \mathrm{ab}$ & $9,00 \mathrm{~b}$ \\
\hline F (Tratamentos) & $11,57 * *$ & $14,48 * *$ & $29,61 * *$ & $6,14 * *$ & $14,63 * *$ \\
Desvio padrão & 25,65 & 17,12 & 2,84 & 24,48 & 6,18 \\
\hline C.V (\%) & 19,41 & 18,34 & 2,28 & 11,59 & 0,37 \\
\hline
\end{tabular}

${ }^{1}$ Médias seguidas de mesma letra na mesma coluna não diferem estatisticamente, a 5\% de significância, pelo teste de Tukey. Para as análises, os dados foram transformados em $(\mathrm{x}+0,5)^{1 / 2}$. ${ }^{2} \mathrm{NIE}=$ número de insetos emergidos; PIE $=$ percentagem de insetos emergidos; PELEA= período de eclosão da larva à emergência do adulto; TMSC = total de massa seca consumida pelos insetos e MSCI= massa seca consumida por inseto.

Quanto ao total de massa de matéria seca seca consumida pelos insetos e de massa de matéria seca consumida por inseto (Tabela 2), podem-se observar diferenças significativas entre os genótipos avaliados. O genótipo Raz 56 foi o menos consumido, quando comparado com os genótipos Dor 391, HF 5465 e Dor 476, em que o consumo foi maior. Os genótipos Arc 2, Raz 49 e Raz 55 ficaram em posição intermediária. Em relação à massa de matéria seca consumida por inseto, o genótipo Raz 59 diferiu de todos os genótipos avaliados, que foram igualmente consumidos, sendo o mais consumido.

De modo geral, os genótipos Raz 56, Arc 2, Raz 55 e Raz 49 apresentaram menores valores de número e percentagem de insetos emergidos, maior período de eclosão, da larva à emergência do adulto, menor de massa de matéria seca consumida total e por inseto, sugerindo portanto, apresentarem resistência dos tipos não-preferência para alimentação e,ou, antibiose. Para o genótipo Raz 59, constatou-se menor número e percentagem de insetos emergidos, maior período de eclosão da larva à emergência do adulto e massa de matéria seca consumida total por inseto em posição intermediária, pois não diferiu dos genótipos mais consumidos, evidenciando apresentar resistência do tipo antibiose.

Por outro lado, os genótipos HF 5465, Dor 391, Dor 476 e IAPAR-MD 806 apresentaram maiores número e percentagem de insetos emergidos e menor período de eclosão, da larva à emergência do adulto, e massa de matéria seca consumida, sendo, considerados, portanto, susceptíveis (Tabela 2).

\section{CONCLUSÕES}

Os genótipos Dor 391, Dor 476 e IAPAR-MD 806 e Raz 59 apresentam resistência do tipo não preferência para oviposição por Z. subfasciatus, em teste com chance de escolha.

Todos os genótipos são igualmente ovipositados pelo inseto, no teste sem chance de escolha.

No teste com chance de escolha, machos e fêmeas são igualmente atraídos por todos os genótipos testados.

Raz 56, Arc 2, Raz 55, Raz 49 e Raz 59 apresentam resistência dos tipos não preferência para alimentação e,ou, antibiose.

\section{AGRADECIMENTOS}

Ao Conselho Nacional de Desenvolvimento Científico e Tecnológico/CNPq, pela bolsa de iniciação científica concedida ao primeiro autor, e de produtividade em pesquisa, ao segundo autor.

\section{REFERÊNCIAS}

Abreu AFB. Pragas e Métodos de Controle (2005) Cultivo do feijão da primeira e segunda safras na região sul de Minas Gerais. Embrapa Arroz e Feijão. Sistemas de Produção, n.6, Versão eletrônica, 2005. Disponível em: <http //www.cnpaf.embrapa.br.htm>. Acessado em: 15 de setembro de 2010.

Acosta-Galegos JA, Quintero C, Vargas J, Toro O, Thome J, Cardona C (1998) A new variant of arcelin in wild common bean, Phaseolus vulgaris L., from southern México. Genetic Resources and Crop Evolution, 45:235-242.

Rev. Ceres, Viçosa, v. 58, n.4, p. 419-424, jul/ago, 2011 
Barbosa FR, Yokohama M, Pereira PAA \& Zimmermann FJP (1999) Efeito da proteína arcelina na biologia de Zabrotes subfasciatus (Boheman 1833), em feijoeiro. Pesquisa Agropecuária Brasileira, 34:1805-1810.

Boiça Júnior AL, Botelho ACG \& Toscano LC (2002) Comportamento de genótipos de feijoeiro ao ataque de Zabrotes subfasciatus (Boheman, 1833) (Coleoptera: Bruchidae) em condições de laboratório. Arquivos do Instituto Biológico, 69:51-55.

Faroni RA \& Silva JS (2008) Manejo de pragas no ecossistema de grãos armazenados. In: Silva JS. (Org.). Secagem e armazenagem de produtos agrícolas. Viçosa, p. 371-406.

Gallo D, Nakano O, Silveira Neto S, Carvalho RPL, De Batista GC, Berti Filho E, Parra JRP, Zucchi RA, Alves SB, Vendramim JD, Marchini LC, Lopes JRS \& Omoto C (2002) Entomologia agrícola, FEALQ, p. 815-898.

Lara F M (1997) Resistance of wild and near isogenic bean lines with variants to Zabrotes subfasciatus (Boheman). I. Winter crop. Anais da Sociedade Entomológica do Brasil, Londrina, 26: 551-560.

Lara FM (1991) Princípios de resistência de plantas a insetos. São Paulo, 336p.

Lorini I (2008) Manejo integrado de pragas de grãos de cereais armazenados. Passo Fundo, Embrapa Trigo, 72p.

Mazzonetto F \& Boiça Junior AL (1999) Determinação dos tipos de resistência de genótipos de feijoeiro ao ataque de Zabrotes subfasciatus (Boheman, 1833) (Coleoptera: Bruchidae). Anais da Sociedade Entomológica do Brasil, 28:307-311.

Mazzonetto F \& Vendramim JD (2002) Aspectos biológicos de Zabrotes subfasciatus (Boh.) (Coleoptera: Bruchidae) em genótipos de feijoeiro com e sem arcelina. Neotropical Entomology, 31: 435-439.

Miranda JE, Toscano LC \& Fernandes MG (2002) Avaliação da resistência de diferentes genótipos de Phaseolus vulgaris à Zabrotes subfasciatus (Boh.) (Coleoptera: Bruchidae). Boletin de Sanidad Vegetal-Plagas, 28:571-576.

Osborn TC, Blake T, Gepts P \& Bliss FA (1986) Bean arcelin, 2: genetic variation, inheritance and linkage relationships of a novel seed protein of Phaseolus vulgaris L. Theoretical and Applied Genetics, 71:847-855.

Paes NS, Gerhardt IR, Coutinho MV, Yokoyama M, Santana E, Harris N, Chrispeels MJ \& De Sá MFG (2000) The effect of arcelin-1 on the structure of the midgut of bruchid larvae and immunolocalization of the arcelin protein. Journal Insect Physiology, 46:393-402.
Pereira PAA, Yokoyama M, Quintela ED \& Bliss FA (1995) Controle do caruncho Zabrotes subfasciatus (Boheman, 1833) (Coleoptera: Bruchidae) pelo uso de proteína da semente em linhagens quase isogênicas de feijoeiro. Pesquisa Agropecuária Brasileira, 30:1031-1034

Procópio SO, Vendramim JD, Ribeiro Junior JI \& Santos JB (2003) Efeito de pós vegetais sobre Acanthoscelides obtectus (Say) e Zabrotes subfasciatus (Boh.) (Coleoptera: Bruchidae). Revista Ceres, 50: 395-405.

Quintela ED (2002) Manual de identificação dos insetos e outros invertebrados pragas do feijoeiro. Santo Antônio do Goiás: Embrapa Arroz e Feijão, 51p.

Ribeiro-Costa CS, Pereira PRVS \& Zukovski L (2007) Desenvolvimento de Zabrotes subfasciatus (Boh.) (Coleoptera: Chrysomelidae, Bruchinae) em genótipos de Phaseolus vulgaris L. (Fabaceae) cultivados no Estado do Paraná e contendo arcelina. Neotropical Entomology, 36:560-564.

Schoonhoven AV \& Cardona C (1982) Low levels of resistance to the Mexican bean weevil in dry bean. Journal of Economic Entomology, 76:567-569.

Schoonhoven AV, Cardona C \& Valor J (1983) Resistance to the bean weevil and the Mexican bean weevil (Coleoptera: Bruchidae) in monocultivated common bean accessions. Journal of Economic Entomology, 76:1255-1259.

Souza EDT, Souza ERB, Veloso AM \& Garcia AH (1997) Nãopreferência para oviposição e alimentação de Zabrotes subfasciatus (Boheman, 1833) (Coleoptera: Bruchidae) em cultivares de feijão (Phaseolus vulgaris L.) portadores de arcelina. Anais da Escola de Agronomia e Veterinária, 57:117-121.

Vieira C, Borém A, Ramalho MAP \& Carneiro JES (2005) Melhoramento do Feijão. In: Borém A. (Ed). Melhoramento de espécies cultivadas. Viçosa, p. 301-391.

Vieira EHN \& Yokoyama M (2000) Colheita, processamento e armazenamento. In: Vieira EHN \& Rava CA. (Ed). Sementes de feijão - Produção e tecnologia. Santo Antônio de Goiás, Embrapa Arroz e Feijão, p. 233-247.

Wanderley VS, Oliveira JV \& Andrade Jr ML (1997) Resistência de cultivares e linhagens de Phaseolus vulgaris L. a Zabrotes subfasciatus (Boh) (Coleoptera: Bruchidae). Anais da Sociedade Entomológica do Brasil, 26:315-320. 